\title{
Pleurectomy in a 10 week old child to control staphylococcal associated pneumothoraces
}

\author{
S LARGE, J WARNER, P GOLDSTRAW \\ From Papworth Hospital, Papworth Everard, Cambridge
}

Staphylococcal pneumonia may be complicated by metastatic abscess, acute endocarditis, meningitis, bronchiectasis, osteomyelitis, pneumatocele, and pyopneumothorax. Staphylococcal pneumonia is a well recognised infection in paediatric practice and about $60 \%$ of cases occur in the first six months of life. Pneumatocele and pyopneumothorax are also more common in infancy. The treatment of staphylococcal pneumonia is mainly medical but occasionally surgical intervention is required. We report the case of a 10 week old child who required pleurodesis by right pleurectomy to control recurrent life threatening pyopneumothoraces.

\section{Case history}

A Caucasian boy born on 14 December 1981 was admitted to his local hospital on 14 January 1982 . He had been tearful and developed poor colour with abdominal distension over the three days before his admission. On examination he was noted to be febrile, listless, and irritable, though well hydrated. Abdominal distension was confirmed and the white blood cell count was found to be $27 \times 10^{9} / 1$ (80\% neutrophils). A tentative diagnosis of meningitis was made. Gram positive cocci were seen in the cerebrospinal fluid after lumbar puncture and treatment was started with chloramphenicol and penicillin parenterally. At this time the chest radiograph was normal.

Three days later the child suddenly became peripherally cool and a chest radiograph showed a large left pneumothorax. After insertion of a chest drain his condition improved and he was transferred to the Brompton Hospital. Soon after arrival his colour again deteriorated and he sustained a respiratory arrest. The chest radiograph showed a right hydropneumothorax. Again his condition improved after insertion of a right sided chest drain. Blood cultures, culture of the cerebrospinal fluid, and pleural aspirate all grew Staphylococcus aureus. His antibiotic regimen was therefore changed to flucloxacillin and gentamicin. On cessation of the air leak the right chest drain was removed but further pneumothoraces occurred on

Address for reprint requests: Mr Stephen Large, House 2, Papworth Hospital, Papworth Everard, Cambridge.

Accepted 8 November 1983 days 7,14 , and 16 and on each occasion further chest drainage was required.

After 25 days he was transferred back to his referring hospital in a satisfactory condition, but five days later he was readmitted to the Brompton Hospital, because his condition had deteriorated (fig 1). A right pneumothorax was drained and he was referred for surgical management of his recurrent right pneumothorax.

On 19 February he underwent right thoracotomy, when loculated pneumothoraces with two air leaks in the anterior surface of the right lung were found. The air leaks were oversewn and a partial parietal pleurectomy was performed. His postoperative course was complicated by bradycardiac episodes unrelated to ventilation. Blood cultures were sterile, and an electroencephalograph and a computed tomography scan of the brain were normal. These episodes became less frequent with time and his recovery was eventually complete. Immunological screening showed no abnormality apart from a low serum IgA concentration $(0.05 \mathrm{~g} / \mathrm{l})$.

The child was discharged well on 31 March 1982, 76 days after his original admission to hospital, and a subsequent chest radiograph showed good expansion of the right lung (fig 2).

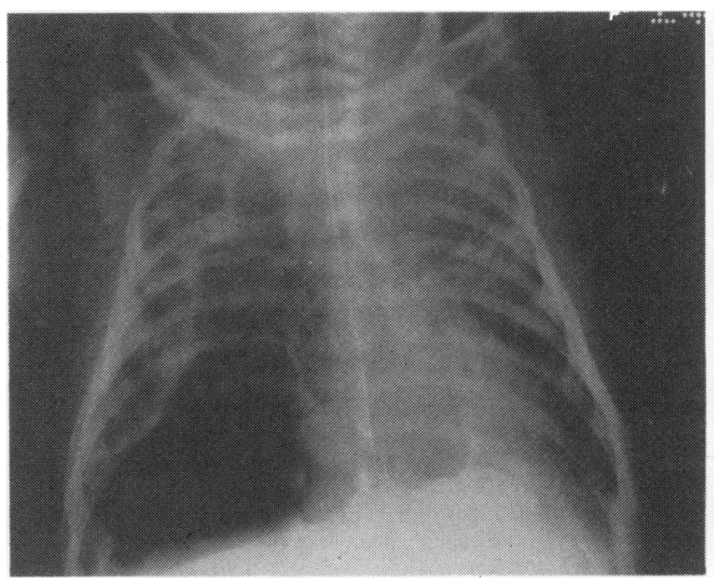

Fig 1. Chest radiograph on readmission with recurrent pyopneumothorax. 


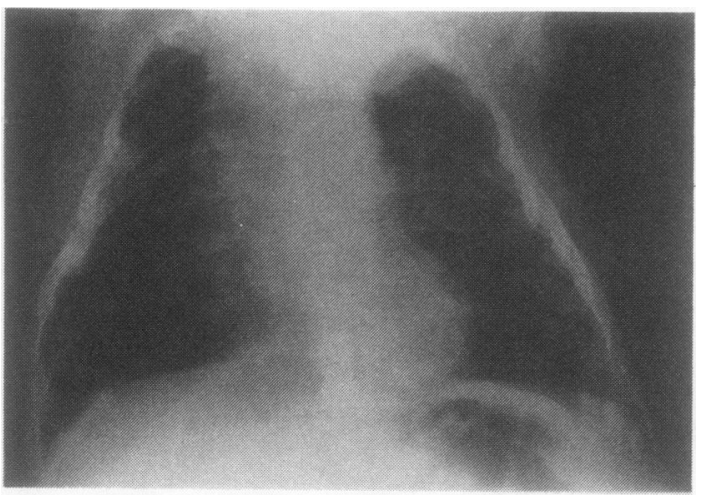

Fig 2 Chest radiograph two months after right apical pleurectomy.

\section{Discussion}

Infants are prone to staphylococcal pneumonia and are more likely than any other age group to develop pneumatoceles and pyopneumothoraces.' Huxtable et al reported a series of 22 children with staphylococcal pneumonia. ${ }^{2}$ All had pleural effusions and 20 of them developed pneumothoraces, which were recurrent in 15. Pneumatoceles occur in roughly $40-60 \%$ of all cases of staphylococcal pneumonia in childhood. These form in the first week of the illness and are reported to disappear by the sixth week. ${ }^{3}$ They probably result from inflammatory destruction of the bronchial epithelium with acinar consolidation, leading to peribronchial abscesses which rupture into a bronchus. The subsequent pneumatocele may enlarge rapidly, but rarely causes cardiorespiratory embarrassment. Intrapleural rupture leads to a pyopneumothorax but even this complication may be managed conservatively, as reported by Schweich and Fierstein. ${ }^{4}$ Nevertheless, surgery has a distinct part to play in the management of some complications of staphylococcal pneumonia. ${ }^{5}$ In the early stages, it may be required for the drainage of a pleural effusion, empyema, or pyopneumothorax, with or without tension, and occasionally direct drainage of a large pneumatocele may be required. Later surgical treatment includes the excision of cysts and drainage of unresolved abscesses or chronic empyemas. Decortication may be necessary after a chronic empyema. These authors note that lobectomy has been described for the control of a tension pneumothorax, ${ }^{5}$ but no description of pleurodesis for the control of staphylococcal associated pneumothorax has been published.

Our patient required surgical intervention for the control of six life threatening episodes of pneumothorax. Five of these occurred on the right side. All were within a space of 33 days and no further episodes occurred after pleurodesis had been performed.

Partial parietal pleurectomy is a well recognised procedure for the control of recurrent or persistent pneumothorax in older age groups. This procedure was first described by Gaensler in $1956 . .^{6}$ It is a safe operation that is associated with minimal measurable changes in ventilatory function. Our case is presented to show that pleurectomy has a role in the management of recurrent pneumothorax of staphylococcal origin in infants and children.

\section{References}

' Gourlay RH. Staphylococcal pneumonia and empyaema in infancy and childhood. Can Med Ass $J$ 1962;87:1101-5.

${ }^{2}$ Huxtable KA, Thicker AS, Wedgewood RJ. Staphylococcal pneumonia in childhood: long term follow up. Am J Dis Child 1964;108:262-9.

${ }^{3}$ Dines DE. Diagnostic significance of pneumatocoele of the lung. JAMA 1968;204:1169-72.

${ }^{4}$ Schweich A, Fierstein J. Staphylococcal septicaemia with recurrent spontaneous pneumothorax. Ann Intern Med 1959; 50:819-27.

${ }^{5}$ Pont ME, Rountree WC. Medical and surgical treatment of staphylococcal pneumonia. Dis Chest 1963;43:176-85.

- Gaensler E. Partial pleurectomy for recurrent spontaneous pneumothorax. Surg Gynec Obstet 1956;102:293-308. 